Ann. Sci. forest., 1976, 33 (4), I99-2 I9.

\title{
MICROFLORE INTERNE DES TISSUS LIGNEUX DE L'ÉPICÉA COMMUN SUR PIED
}

\author{
I. - INVENTAIRE DE I,A MTRROFLORE NATUREI,I,E \\ C. DELATOUR \\ aver la collaboration technique de Gilberte Svzvestra \\ Laboratoire de Pathologie forcstizre, \\ Cente national de Recherches forestieves, I. N.R.A. \\ Chanpenoux, 5t280 Seichamps
}

\section{RÉSUMÉ}

Afin de pouvoir étudier ultérieurement son influence sur la propagation du $F$. annosus a l'intérieur des arbres vivants (Picea abies), l'auteur a cherché a mettre en évidence la microflore accompagnatrice de l'altération. Cent arbres ont été examinés à $1,30 \mathrm{~m}$ puis 49 d'entre eux à $0,30 \mathrm{~m}$ de hauteur; 5 arbres furent ensuite l'objet d'observations plus détaillées. L'étude des arbres sur pied a été faite à l'aide de la tarière de Pressler ; le dépót des carottes-échantillons sur milieu nutritif gélosé permet l'obtention des micro-organismes qu'elles hébergent. Outre le $F$. annosus, furent isolés : Mollisia sp., Nectria fuckeliana var. fuckeliana, Ascocoryne sarcoides et, moins fréquemment des représentants des genres Scylalidium, Trichoderma, des mucorales, bactéries, etc. guếs :

Suivant leur disposition par rapport au bois sain ou altéré, trois groupes ont pu ètre distin-

1. les espèces liées préférentiellement au bois sain : Mollisia sp., N. fuckeliana var. fuckeliana;

2. les espèces liées au bois altéré comme au bois sain : $F$. annosus, $A$. sarcoides ;

3. les espèces liées exclusivement au bois altéré : Scytalidium, Trichoderma, bactéries, etc.

Les espèces du groupe 3 se rencontrent plus fréquemment à o, $30 \mathrm{~m}$ et sont caractéristiques de stades d'évolution avancés de l'altération desquels le $F$. annosus peut être éliminé,

Le groupe I est constitué de champignons qui précédent le $F$. annosus mais dont le rôle ne peut être défini par la simple observation en nature. La présence du Mollisia sp. à ce niveau est une observation inédite.

Le groupe 2 est celui des champignons d'altération, coci est connu pour le F. annosus mais demande à étre précisé pour 1'A. sarcoides.

\section{INTRODUCTION}

L'Épicéa commun (Picea abies (L.) KARstês) est une essence très répandue en France ainsi qu'en Europe. Sa plasticité a permis son installation dans des conditions écologiques très variées où il s'est montré n'être l'objet que de peu d'altérations 
d'ordre parasitaire. Sur le plan pathologique, il subit, parfois de façon intense, les atteintes du Fomes annosus (FR.) CoOkT, agent d'une pourriture de cour (PERRIN et Disatouk, 1975). Il nous a été donné de faire le point des connaissances acquises sur ce problème (DEI,ATOUR, I972) ; rappelons que, pour un arbre donné, l'attaque débute généralement au niveau racinaire et se développe en direction du tronc, la pourriture progresse done dans le fût de bas en haut.

Ia propagation interne peut être rapide, $\mathrm{I}, 3 \mathrm{~m}$ en deux ans d'après RENNERFEr,T (I947), mais elle peut rencontrer un certain nombre de freins. SHarN (I97I) a montré qu' une a zone de réaction "située dans l'aubier limite la progression diamétrale. Verticalement, la pourriture peut atteindre des hauteurs importantes $(7 \mathrm{a} 8 \mathrm{~m}$, Anonyme, I924, Maraite, I965) mais il est fréquent que la progression ralentisse ou même s'arrête plus tòt. Ceci a été observé de façon évidente en Suède par RENNIERFEIT (cité par RICARD, I970) dont nous avons eu l'occasion de visiter la placette expérimentale grâce à l'amabilité de J. RICARD : chez plusieurs arbres la colonne d'altération ne progresse plus depuis plusieurs années arrêtée à des hauteurs variables. Nous avons nous-même (PLRRIN et DÉ.ATour, sous presse) observé des phénomènes comparables lors de l'étude de la configuration des colonnes d'altération.

Plusieurs hypothèses peuvent être envisagées pour expliquer cela :

1. variation dans le comportement du champignon,

2. changement intervenant dans le milieu ligneux,

3. compétition avec d'autres micro-organismes.

Le seul fait qui corresponde à 1'hypothèse I a été rapporté par MARAITE (I965) qui signale qu'à partir des altérations avancées chez l'Épicéa (stades I et 2 de l'auteur), on isole des souches de $F$, annosus à croissance lente alors qu'à partir de la zone où l'altération n'est qu'à son début (stade 3), les souches ont une croissance rapide. Cette observation n'a jamais été reprise depuis, elle signifierait cependant que dans un même arbre, le $F$, annosus peut présenter des variations de comportement. Ces variations mériteraient d'être étudiées en prenant en compte notamment l'aptitude à la colonisation endophyte. L'hypothèse 2 est basée en particulier sur le fait que le $F$. ansosars est limité dans sa croissance par l'aubier de l'arbre qui réagit en formant une zone de réaction (SHars, 197 I). Il découle de cette observation que le bois colonisable par le $F$. annosus dans un arbre donné forme un cóne interne de hauteur définie dont nous ignorons cependant les règles d'accroissement. D'autre part, des facteurs stationnels ou individuels (phénomène de dominance, état physiologique) influencent probablement le caractère de résistance de l'aubier dont dépend la hauteur colonisable.

Notre travail a eu pour but d'étudier la troisième hypothèse, certaines observations antérieures montraient l'existence de champignons et de bactéries dans le bois sain d’arbres sur pied; pour les résineux, les observations avaient surtout été faites chez des Pins et restaient beaucoup plus fragmentaires chez l'Épicén.

Chez un épicéa nord-américain, ErHTrmos (I956) avait trouvé l'Ascocoryne sarcoides (JACQ. ex GRAY) Groves et Wilson dans le bois de cour de sujets sains, Rot, L-HANSEN (I962) puis ShaIN (I971) avaient mis en évidence l'existence du Nectria fuckeliana nooth var. fuckeliana вoorH dans le bois sain de Picea abies puis RICARD (1970) avait isolé I'A. sarcoides et Cephalosporium s1. au niveau des colonnes d'altération stabilisées. 
La question du rôle de ces champignons dans d'éventuels processus d'altération ou vis-à-vis d'agents lignivores dûment reconnus, était évoquée par divers auteurs.

Il s'agissait donc pour nous, par un inventaire le plus complet possible, de préciser l'existence de la microflore accompagnatrice du $F$. annosus dans les troncs d'épicéas attaqués afin de pouvoir étudier ultérieurement le rôle qu'elle joue dans la progression endophyte du parasite.

\section{I. - MATÉRIEL ET MÉTHODES}

\section{1. - Placettes Etudies}

L'inventaire systématique de la microflore a eu lien dans une placette d'Épicéa commur. (6o ans environ) attaquée par le Fomes annosus, située en forét communale de Liffol-le-Grand (Vosges, 88).

Les échantillons suivants ont été étudiés :

a) Cent arbres pris de façon systématique ont été sondés à $1,30 \mathrm{~m}$ (série totale),

b) Au vu des résultats obtenus, 49 arbres ont été retenus parmi les précédents pour sondages à $0,30 \mathrm{~m}$ (série complète). Ont été choisis préférentiellement, les arbres chez lesquels le $F$, annosus était absent à $1,30 \mathrm{~m}$ ou ceux qui présentaient, à ce niveau, une microflore particulièrement intéressante,

c) enfin, cinq arbres ont fait l'objet d'une étude plus précise de la répartition de la microflore : un arbre a été étudié sur pied car trop volumineux, les quatre autres ont été abattus et étudiés au laboratoire.

\section{2. - Prelevement et isolement chez les arbres sier pied}

Les prêlèvements ont été effectués en forèt à la tarière de Pressler (diamètre intérieur $0,5 \mathrm{~cm}$ ) suivant le mode opératoire suivant :

- la tarière et l'extracteur sont trempés dans l'alcool à 76 p. 100 puis égouttés rapidement au moment de leur emploi. Le trempage de la tarière s'effectue grâce à une fiole cn polyéthylène souple remplie d'alcool dont le bouchon est muni d'un tube plongeur; une simple pression sur les parois de la fiole provoque la montée de l'alcool dans le tube et donc l'immersion de la tarière si elle y a été préalablement introduite,

- au niveaa choisi pour le prélèvement, une rondelle d'écorce est enlevée atu scalpel et la plaie tamponnée à l'alcool,

- le sondage est effectué de façon perpendiculaire à larbre pour atteindre le coeur.

- la carotte-échantilton est retirée rapidement et déposée dans un tube à essai stérile bouché au coton,

- au laboratoire, les carottes-échantillons sont déposées sur gélose au malt (3 p. 10o) en boite de Petri après un rapide passage à la flamme (Gillerte SYLvestux, 1974). Les carottes de plus de $8 \mathrm{~cm}$ de longueur sont fractionnées en plusicurs tronçons qui sont alors soigneusement repérés. Les boites de Petri sont incubées en étuve ou dans les conditions du laboratoire. Après observations journalières, la lecture finale se fait au bout d'une dizaine de jours lorsque la microflore est suflisamment développée.

II est à souligner que l'élimination de l'écotce au scalpel sur le terrain et le flambage rapide au laboratoire sont les deux conditions qui, en plus des règles ordinaires d'asepsie, permettent d'obtenir des prélèvements propres.

\section{3. - Isolement dans les arbres abattus}

Les portions de trone à étudier sont découpées à la tronçonncuse en sections de go cm de long et rapportées au laboratoire, Leur écorce est brossce vigoureusement sous l'eau courante. Lorsque les rondins sont secs, ils sont sciés en long par moitié ì la scierie, les surfaces de coupe sont nettoyées de la sciure avec une brosse propre. Les faces sur lesquelles seront opérés les 
prélèvements sont exposées aux rayons ultra-violets au laboratoire après que les points de prêtèvements aient été marqués au crayon. Aprés 15 à 30 minutes d'exposition à $10-20 \mathrm{~cm}$ du tube, les zones intéressantes sont recouvertes de bandes de papier stériles que l'on agrafe et qui ne sont enlevées qu'au fur et à mesure des prélèvements pour éviter leur recontamination. Les prélèvements sont effectués dans la chambre à isolements grâce à une gouge de sculpteur à gorge demi-ronde de $3 \mathrm{~mm}$ de dismètre. Les fragments de bois $(0,3 \mathrm{~cm}$ environ en tous sens $)$ sont déposés en boites de Petri sur gélose au malt 3 p, 100.

Cette technique qui donne des conditions d'asepsie excellentes, est, a notre avis, beaucoup plus facile d'emploi que celle développée par PAwsEY at al. (1974) qui nécessite un trempage des rondins dans le chlorure mercurique 0,1 p. 100 suivi d'un rinçage et d'un égouttage.

\section{2. - RÉSULTATS}

\section{I. - Les espèces isolées}

Ainsi que nous l'expliquerons ultérieurement, nous avons porté notre attention en priorité sur les espèces colonisatrices primaires du bois et non pas sur celles qui, secondaires, peuplaient abondamment le bois déjà largement altéré. Compte tenu de cette orientation, nous avons délibérément regroupé les espèces secondaires sous la rubrique « autres micro-organismes » sans avoir cherché à pousser leur identification ; parmi ces espèces, le genre Scytalidium a cependant été traité à part à cause de ses propriétés antagonistes potentielles en milieu ligneux, nous y reviendrons.

\section{II. Fomes annosus (FR.) COOKE.}

Ce basidiomycète est très facilement identifiable grâce à sa forme imparfaite Gidocephalum meineckella (HOL.sox)) DANK. caractéristique; sur malt-agar 3 p. Ioo, sa croissance radiale est de $5^{\circ} \mathrm{mm}$ en 8 jours à $26^{\circ} \mathrm{C}$. Il est sulfisamment classique pour que l'on ne s'y arrête pas.

\section{12. Mollisia SP.}

Cette espèce, que nous verrons être un des éléments les plus fréquents de la microflore interne de l'Épicéa, ne développe en culture que sa forme imparfaite appartenant au genre Phialophora.

La colonie est d'abord hyaline puis se colore en brun verdâtre évoluant finalement vers le noir. Le mycélium est d'abord apprimé sur la gélose et intramatriciel, puis un feutrage aérien parfois très abondant peut se développer, de couleur grise puis nuancée de rouge avec l'âge. La croissance radiale est lente ( $15 \mathrm{~mm}$ en 8 jours à $\left.26^{\circ} \mathrm{C}\right)$. In vitro, la production d'un pigment rouge brique semble être une caractéristique de ce champignon : nuance rouge des vieux hyphes, coloration rouge brique du milieu gélosé dès le quinzième jour de culture, cristaux de même couleur qui apparaissent dans les vieilles cultures, constitués de paillettes très effilées, le plus souvent disposées en faisceaux ou en étoiles. Nous avons tout lieu de penser que ce pigment est de la mollisine ou s'y apparente (MANGENot, GaMs, communications personnelles).

La fructification imparfaite se rattache donc au genre Phialophora ; elle se développe dès le premier mois de culture. Les phialides sont disséminées le long du mycélium, isolées ou groupées par 2 ou 3 , dimensions : $7^{-13} \times 6-3 \mu$. Les conidies sont 
hyalines, monocellulaires, rondes ou ovoīdes, $\mathrm{I}, 4^{-1}, 8-2,4 \times \mathrm{I}-\mathrm{I}, 4^{-2} \mu$, elles demeurent généralement groupées en tête des phialides. On peut voir également apparaitre des glomérules blancs puis jaunâtres mesurant jusqu'à $o, 1 \mathrm{~mm}$ de diamètre constitués d'hyphes renflés, agglomérés, dont la structure peut rappeler certains éléments extérieurs des apothécies. Nous n'avons pas pu rattacher cette forme Phialophora à une espèce connue.

Nous avons obtenu la forme parfaite Mollisia SP. (Discomycète-Hélotiale) en mai I975 sur des bûchettes d'Épicéa inoculées puis laissées l'hiver en nature. Des réisolements à partir d'ascospores nous ont donné des souches identiques à celle de départ. L'étude que nous avons effectuée de la fructification parfaite ne nous a pas permis de conclure à une espèce précise, le genre Mollisia demeurant encore confus malgré l'important travail de Le GAL et MANGenot (I958, I959, I96r, I966). Les principales caractéristiques de notre Mollisia sont les suivantes : apothécies arrondies jusqu'à $0,5 \mathrm{~mm}$ de diamètre dans nos exemplaires, marge régulière, stipe court de couleur brune, hyménium blanc, d'abord concave puis convexe à complète évolution, apothécie prenant sur le sec une couleur plus terne, jaunâtre. L'hyménium est constitué des éléments classiques chez les Mollisia : paraphyses filiformes, plus longues que les asques, asques à pore teinté en bleu par 1'iode, ascospores fusiformes, bisériées, monocellulaires ou bicellulaires $6,5-7,5-8,5 \times$ I, $3-1,6-1,9 \mu$.

La littérature ne nous renseigne en aucune façon sur ce Mollisia sp. Aucun représentant de ce genre n'a jamais été identifié à notre connaissance dans les tissus internes de l'T́picéa vivant ou même d'autres résineux. Des mentions de Phialophora SPp. ont été faites pour l'Épicéa : Phialophora SP. (MaraItE, 1965 ; Schönhar, I969; Pechmann, Aursess, I971), Phialophora fastigiala (Pechmann, Aufsess, I971; Kato, I967), Phialophora malorum (Pechmans, Aufsess, 1971). Ces espèces ont toutes été isolées au niveau de bois altérés, ce qui correspond assez pau à la localisation de notre Mollisia sp. comme nous le verrons.

\section{13. Nectria fuckeliana воотн VAR. fuckeliana воOтн.}

Ce champignon se caractérise en culture par son abondante fructification microconidienne de type Acremonium (= Ciphalosporium) et par une coloration jaune orangé fréquente chez les Nectria. La croissance radiale est de $\mathrm{I}_{3} \mathrm{~mm}$ en 8 jours à $26^{\circ} \mathrm{C}$. La forme macroconidienne appartient au genre Cylindrocarpon, elle apparaît sur milieu gélosé ou sur bûchettes de bois, les macroconidies présentent jusqu'à 7 cloisons ( $30 \mu$ de long). Des ébauches de périthèces peuvent apparaitre sur bûchettes. Nous avons, d'autre part, obtenu une souche aux mêmes caractéristiques morphologiques et micrométriques à partir d'ascospores du Nectria fuckeliana BoTH VAR. fuckeliana BooTH récolté en bordure de chancre sur un épicéa mort (Rambervillers, 1969). Notre champignon en culture est conforme à la description faite par Rol,-HANSEN (1962) pour le Nectria cucurbitula sENSU WOLLENWEBER dont la forme macroconidienne est le Cylindrocarpon cylindroides WoI.LENW. VAR. tenuc WOLLENw. Ėn I966, BooTn donnait cette dernière espèce comme forme conidienne du Nectria fuckeliana BoотH var. fuckeliana BooтH. Cephalosporium sP., Cylindrocarpon cylindroides VAR. tenué, Nectria cucurbitula et $N$. fuckeliana ont été fréquemment mentionnés antérieurement dans les tissus ligneux de l'Épicéa. Pour Cephalosporium SP. nous ne pouvons avoir aucune certitude d'identification spécifique en l'absence d'une comparaison de cultures mais pour $N$. cucurbitula, il est probable 
qu'il s'agisse généralement du $N$. fuckcliana var. fuckeliana comme nous l'avons vu pour celui étudié par RoL,L-HANsEN (Ig62).

\section{I4. Ascocoryne sarcoides (JAce, ex GRAy) Groves et Wilson.}

Ce champignon se caractérise par ses cultures à l'aspect gras et de couleur rose vineux souvent intense. Croissance radiale de $18 \mathrm{~mm}$ en 8 jours à $26^{\circ} \mathrm{C}$. Des conidies se développent abondanment à l'extrémité de conidiophores ramifiés et demeurent agglomérées en masses muqueuses. Comme le note BERTHEr (I964), les conidies sont de deux types, les unes allongées, bacilliformes, les autres arrondies. Nos mesures sont les suivantes:

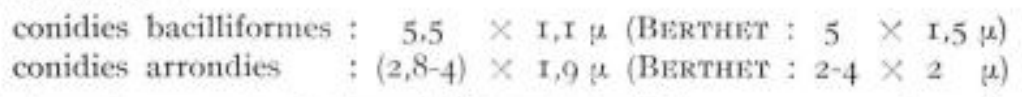

Ainsi que le rapporte également Bங்RTuros (I964), nous avons observé, dans les cultures âgées, des formations charnues globuleuses ou allongées (jusqu'à I cm), parfois ramifiées, rose vineux, tout à fait comparables à celles qui se développent dans la nature sur les souches au voisinage de la forme ascosporée. Ces formations constituent la forme imparfaite Pirobasidium sarcoides (JACQ.) новнN. de l'Ascocoryne sarcoides (JACQ. ex GRAY) Groves et Wilson (= Coryne sarcoides (JACQ.) TUL..) Ce champignon a été mis en évidence dans le bois de résineux pour la première fois par Etheridge, (r953) chez Picea glauca, P. cngclmanni et P. mariana au Canada. Nous avons observé la forme ascosporée, parfois en abondance, dans les peuplements d'épicéas, le plus souvent sur les souches.

Les mentions d'A. sarcoides ou * $A$. sarcoides complex " sont nombreuses dans la littérature, aussi bien sur feuillus que sur résineux, ce champignon est donc parfaitement ubiquiste. Ses hôtes résineux connus appartiennent aux genres : Picca, Abies, Pinus, Pseudotsuga, Tsuga, Larix (Frrhermbli, I97o).

\section{I5. Scytalidium SP.}

Plusieurs souches appartenant à ce genre ont été isolées. Le genre, créé par Pesante (1956), appartient aux Dématiacées et se caractérise par la présence d'arthrospores caténées hyalines et de chlamydospores sombres en chapelets. Nos souches ne se rattachent pas toutes de façon évidente aux espèces actuellement décrites. Une étude systématique du genre est en cours au laboratoire indépendamment du présent travail.

Des observations préliminaires nous ont permis de distinguer detux groupes :

I. Groupe * noir a (proche de S. lignicola PESANTE), apparition précoce des chlamydospores régulièrement centrifuges sous forme de rayons ; absence de pigmentation (jaune) du milieu de culture, pas d'antagonisme (1) à distance vis-à-vis du $F$. annosus mais envahissement de celui-ci ; croissance de $37 \mathrm{~mm}$ en 8 jours à $26^{\circ} \mathrm{C}$

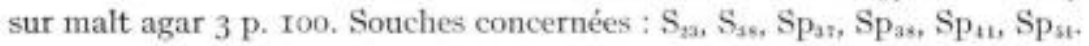

2. Groupe * blane n (proche de $S$. album et $S$. aurantiacum), apparition irrégulière des chlamydospores, par plages: irisations autour de l'implant, pigmentation jaune du milieu; antagonisme à distance vis-à-vis du $F$. annosus; croissance de $3^{\circ} \mathrm{mm}$ en 8 jours à $26^{\circ} \mathrm{C}$ sur malt agar $3 \mathrm{p}$. I00. Sotuches concernées : $\mathrm{S}_{4}, \mathrm{~S}_{36}, \mathrm{Sp}_{30}$.

(1) Antagonisme observé par confrontation sur milieu gélosté en boites de Petri. 
Des Scytalidium ont été mentionnés au niveau du bois altéré chez Picea abies (Maraite, 1965; Klingstrom et Bayer, 1965; Pechmann et Aursess, i97r), chez Pinus banksiana (Basham, I966) et chez des feuillus (notamment Pesante, 1956), il en a été également mentionnés dans des copeaux et dans le sol.

Certains représentants du genre Scylalidium ont montré des propriétés antibiotiques intéressantes notamment vis-à-vis du $F$. annosus (Kt,1NGstrom et al., 1965; Strunz et al,, I972, Striwitr. et al., I973). In situ, Ricard (1975) semble avoir obtenu un effet curatif efficace contre Poria carbonica en inoculant Scylalidium SP. (FY) au pied de poteaux téléphoniques de Douglas alors qu'ils présentaient un début d'attaque.

\section{I6. Autres micro-organismes.}

Comme nous l'avons déjà signalé, ce groupe ne se rencontre, ainsi que le genre Scytalidium d'ailleurs, qu'au niveau des pourritures avancées ; parmi ces organismes, figurent notamment le genre Trichoderma, des Mucorales, des bactéries; nous avons même observé des nématodes dont certains au moins sont l'Aphelenchoides parietimus (').

\section{2. - Les populations de micro-organismes ct leur répartition dans l'hôte}

\section{21. Résultats bruts.}

I. tableau I récapitule les résultats obtenus sur la totalité des sondages et isolements effectués à $\mathrm{I}, 30 \mathrm{~m}$ et à $0,30 \mathrm{~m}$. La distinction a été faite dès ce niveau entre les isolats obtenus à partir du bois sain et ceux à partir du bois altéré. Nous avons inclus sous le terme * bois altéré * toute anomalie allant de la simple coloration aux modifications structurales avancées. Un classement a été fait en fonction de l'altération d'une part et de la présence du $F$, annosus d'autre part ; les numéros I à 6 (n d'ordre) sont les arbres sains indemnes de micro-organismes, les numéros 7 à 80 , les arbres sains ou non hébergeant le $F$. annosus, les numéros $8 \mathrm{I}$ à roo, les arbres sains ou non présentant des micro-organismes mais pas le $F$. annosus.

Si l'on ne considère que le $F$. annosus dans la série où il est présent et compte tenu de ce que nous savons du caractère lignivore de ce champignon, nous devons admettre que nous avons ici divers stades d'évolution ou faciès de son attaque. Le classement que nous avons adopté nous permet de reconstituer l'attaque de la façon suivante :

$I^{\circ}$ Le $F$. annosus colonise le bois sain ou nous le trouvons d'abord au pied $\left(\mathrm{n}^{\circ} 7-8\right)$ puis à $1,30 \mathrm{~m}\left(\mathrm{n}^{\circ} 9\right.$-10).

$2^{\circ}$ Le $F$. annosus dégrade le bois colonisé d'abord au pied (n० 12 à I6) puis à $\mathrm{I}, 30 \mathrm{~m}\left(\mathrm{n}^{\prime \prime} 57,64\right.$ à 73$)$. Le phénomène d'altération se développe donc à la suite de la colonisation fongique mais avec un certain décalage. Ce décalage est variable, plus grand chez les numéros 9, ro que chez les numéros I5, I6.

$3^{\circ} \mathrm{A}$ un stade plus avancé, le $F$, annosus peut sans doute disparaitre du bois qu'il a colonisé mais il n'est pas possible d'avoir de certitude sur la colonisation antérieure : il est absent au pied chez les numéros 75 à 77 , à $1,30 \mathrm{~m}$ chez les numé-

(2) Détermination Station de Recherches sur les Nèmatodes I.N.R.A., Antibes. 
Resultats brats, Microflote inter Rough results. Interual micr

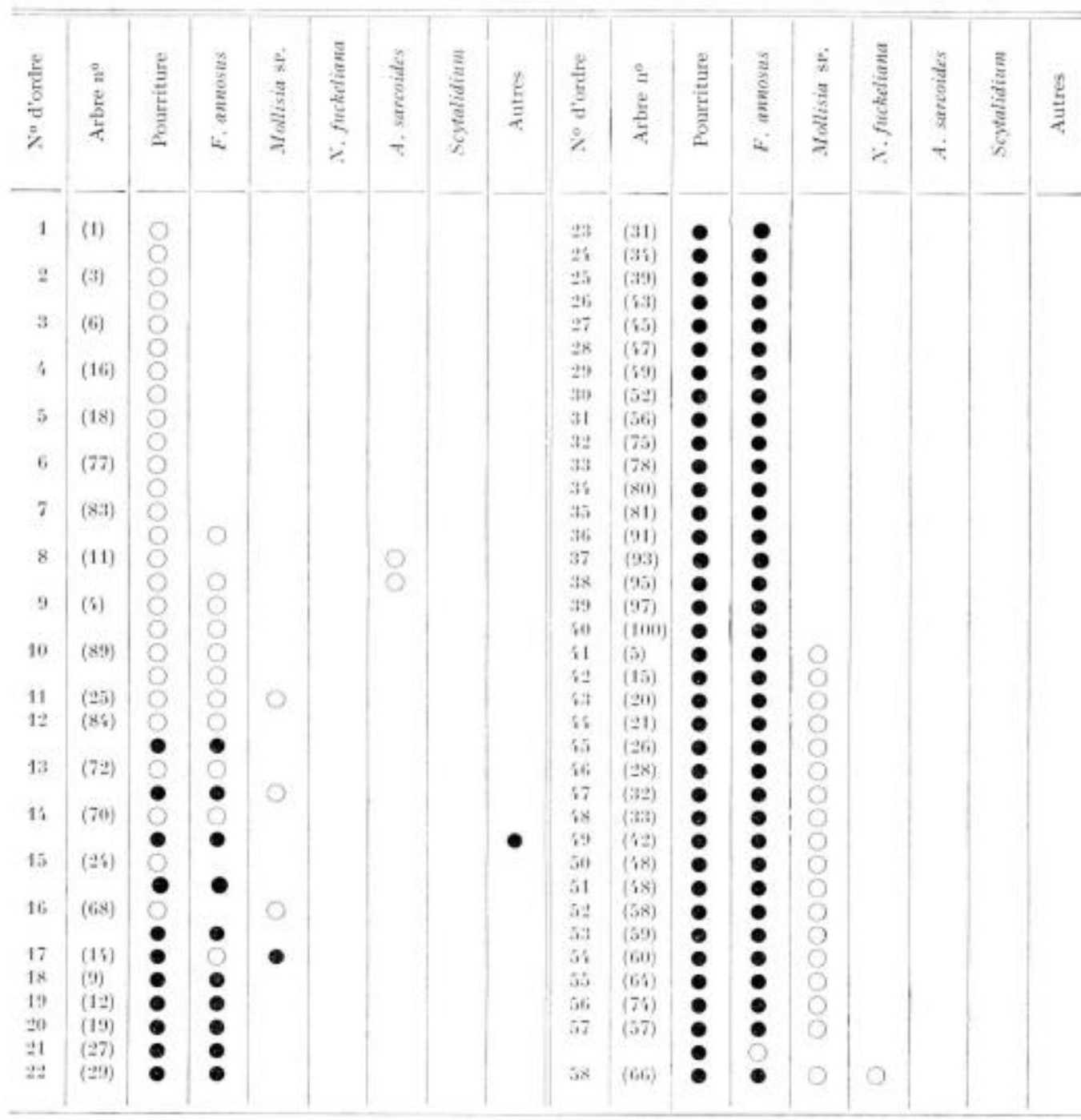

Bois sain ou isolement obtenu dans din bois sain.

(Sound wooul or strain from sound wood).

- Hois altèré ou isolement obtenu dans du bois altêré.

(Decayed nowd of strain from decayed anod).

Lorsque pour un arbre deux lignes sout figurixs, la supericure est celle du sondage a $1,30 \mathrm{~m}$; l'inféricure, cell

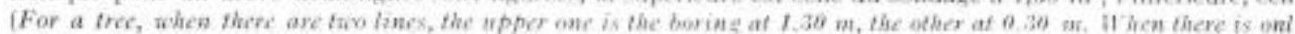


$\mathbf{U}$

lée par sondages arbre par arbre

ra obfained by boring tress

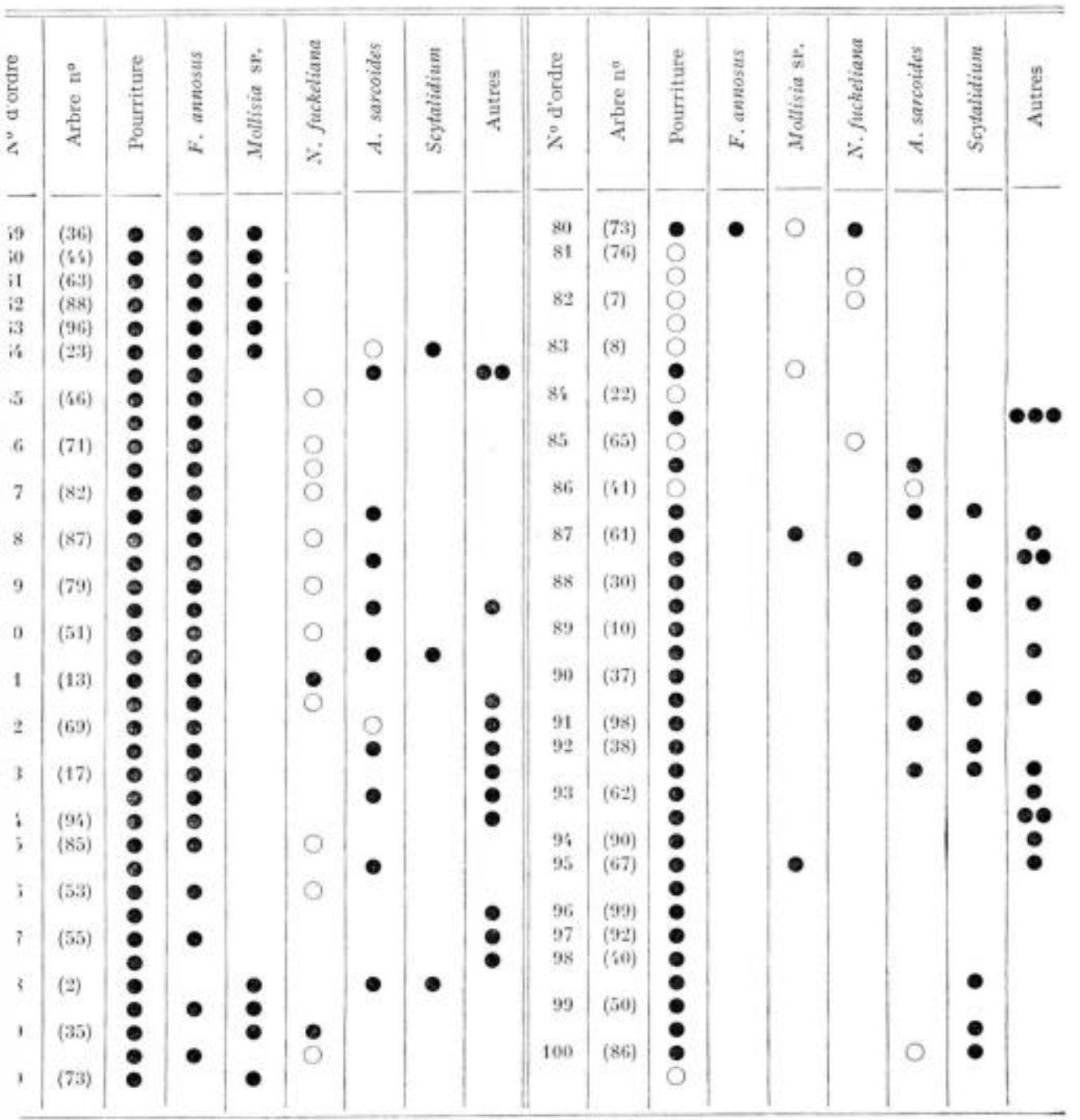

indage à $0,30 \mathrm{~m}$. Lorsgu'une seule ligne est figurée, il s'agit d'un sondage a $1,30 \mathrm{~m}$. ine, it is a boring at $1.30 \mathrm{~m}$ ) 
ros 78 à 80 ; dans la série $8 \mathrm{r}$ à Ioo, il est possible qu'il y ait quelques cas de disparition du $F$. annosus aux deux niveaux.

Ce schéma qui ne prend en considération que le $F$. annosus et l'altération du bois correspond bien à ce que l'on connait de l'évolution de ce champignon chez l'Épicéa ; cependant, nos observations montrent que dans de très nombreux cas, d'autres micro-organismes sont également présents dans les trones; dans un cas (n० 64), nous avons obtenu jusqu'à quatre espèces différentes à un même niveau de sondage.

Examinons successivement les espèces en cause :

Le Mollisia SP, apparait très généralement associé au $F$. annosus (série $4 \mathrm{I}$ à 64 notamment) et se situe plus fréquemment dans le bois sain. Le N. fuckeliana var. fuckeliana présente les mêmes tendances. L'A. sarcoidés est localisé nettement dans le bois altéré mais peut se trouver aussi dans le bois sain (n ${ }^{\circ} 8$ notamment) ; il est particulièrement abondant dans la série 8 I à roo de laquelle le $F$. annosus est absent. Scytalidium SP. et la catégorie " autres o ne se trouvent que dans le bois altéré et sont surtout abondants dans la série $8 \mathrm{I}$ à $\mathrm{r} 00$.

Ces observations faites à la simple lecture des résultats bruts ne nous permettent sans doute de voir que les caractéristiques les plus évidentes de cette microflore " accompagnatrice $»$ du $F$. annosus. Nous avons procédé à son analyse plus détaillée et en rendons compte ci-après.

\section{22. Répartition de la microflore dans l'höte.}

a) En fonction du niveau de sondage (tabl, 2).

Sans faire de distinction entre le bois sain et le bois altéré, nous constatons qu'à $\mathrm{I}, 30 \mathrm{~m}$, dans la w série totale ", les micro-organismes sont présents avec des fréquences diftérentes, le $F$. annosus venant largement en tête. A ce même niveau, nous voyons que dans la " série complète n, les fréquences ne sont pas les mêmes, ceci est dû au biais que nous avons introduit par notre choix lors de l'établissement de cette série; cependant, nous pouvons ici comparer les niveaux $1,30 \mathrm{~m}$ et $0,30 \mathrm{~m}$. De cette comparaison, il ressort que Mollisia SP. et $N$. fuckeliana var. fuckeliana sont nettement plus fréquents à $\mathrm{I}, 30 \mathrm{~m}$ qu'au pied et que l'inverse se produit pour $A$. sarcoides et la catégorie " autres $x$ qui, au pied, se classe alors juste après le $F$, annosus.

\section{b) En fonction de l'altération (tabl. 3).}

Dans chaque sondage effectué aux niveaux altérés, nous pouvons distinguer, en plus de la partie de bois altéré, une partie de bois sain de telle sorte que, même dans ces sondages, nous pouvons obtenir des espèces issues de bois sain. A partir de la totalité des sondages, chaque espèce peut donc être caractérisée par sa fréquence d'apparition dans le bois sain d'une part et dans le bois altéré d'autre part; le tableau 3 montre les résultats obtenus à cet égard; il ressort que, pour chaque espèce, la fréquence dominante se rencontre toujours dans la même catégorie (bois sain ou altéré) quel que soit le niveau envisagé ( $\mathrm{r}, 30 \mathrm{~m}$ ou $0,30 \mathrm{~m})$. Ainsi, nous pouvons distinguer trois groupes à comportements différents :

I. Les espèces liées préférentiellement au bois sain : Mollisia SP., N. fuckeliana vAR. fuckeliana; 


\section{TABI,EAU 2}

Répartition des especes a $0,30 \mathrm{~m}$ et aे $1,30 \mathrm{~m}$

Distribution of the species at $0.30 \mathrm{~m}$ and $1.30 \mathrm{~m}$ levels

\begin{tabular}{|c|c|c|c|c|c|c|}
\hline & \multirow{2}{*}{\multicolumn{2}{|c|}{$\begin{array}{l}\text { Série totale } \\
\text { (Tatal series) } \\
\text { 1,30 m }\end{array}$}} & \multicolumn{4}{|c|}{ Série complète (Couplete series) } \\
\hline & & & \multicolumn{2}{|c|}{$1,30 \mathrm{~m}$} & \multicolumn{2}{|c|}{$0,30 \mathrm{~m}$} \\
\hline & Nombre & P. 100 & Nombre & P. 100 & Nombre & P. 100 \\
\hline$F$, asnuasus $\ldots \ldots \ldots \ldots \ldots \ldots$ & 67 & 50,7 & 19 & 32,2 & 23 & $3 t, 0$ \\
\hline 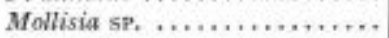 & 31 & $2: 1,5$ & 8 & 13,5 & 6 & 5,6 \\
\hline N. fuckeliana var. fuckeliana .. & 14 & 10,6 & 13 & 22,0 & 5 & 6,8 \\
\hline A. sarcoides, . . . . . . . . . . . . & 10 & 7,6 & 4 & 15,2 & 17 & 23,0 \\
\hline Scytalidium sr. ........... & 4 & 3,0 & 4 & 6,7 & 5 & 6,8 \\
\hline Autres (others) $\ldots \ldots \ldots \ldots \ldots$ & 6 & 4,6 & 6 & 10,4 & 20 & 27,0 \\
\hline Toral ............... & 132 & 100 & 59 & 100 & 74 & 100 \\
\hline
\end{tabular}

\section{TABLEAU 3}

Répartition des espèces entre le bois sain et le bois altéré

Distribution of the species in the sound sood and in the decayed toood

\begin{tabular}{|c|c|c|c|c|c|c|c|}
\hline & & \multicolumn{3}{|c|}{$\begin{array}{c}1,30 \mathrm{~m} \\
\text { Série totale } \\
\text { (Total seriés) }\end{array}$} & \multicolumn{3}{|c|}{$0,30 \mathrm{~m}$} \\
\hline & & $\begin{array}{l}\text { Bois sain } \\
\text { (sound } \\
\text { sood) }\end{array}$ & $\begin{array}{c}\text { Bois altéré } \\
\text { (decayed } \\
\text { wood })\end{array}$ & Total. & $\begin{array}{l}\text { Bois sain } \\
\text { (sound } \\
\text { wood) }\end{array}$ & $\begin{array}{c}\text { Bois altérét } \\
\text { (decayed } \\
\text { mond) }\end{array}$ & TотмL \\
\hline \multirow{2}{*}{ F. annosus } & Nbre & 7 & 60 & 67 & 4 & 19 & 23 \\
\hline & $\%$ & 10,5 & 89,5 & 100 & 17,4 & 82,6 & 100 \\
\hline \multirow{2}{*}{ Mollisia sP. } & Nbre & 19 & 12 & 31 & 3 & 1 & 6 \\
\hline & $\%$ & 61,2 & 38,8 & 100 & 75,0 & 95,0 & 100 \\
\hline \multirow{2}{*}{ N. fuckeliana } & Nbre & 12 & 2 & 14 & 4 & 1 & 5 \\
\hline & $\%$ & 85,7 & 14,3 & 100 & 80,0 & 20,0 & 100 \\
\hline \multirow{2}{*}{ A. sarcoiles } & Nbre & 4 & 6 & 10 & 1 & 16 & 17 \\
\hline & $\%$ & 40,0 & 60,0 & 100 & 5,9 & 94,1 & 100 \\
\hline \multirow{2}{*}{ Scytalidium sP. } & Nbre & 0 & 4 & 4 & 0 & 5 & 5 \\
\hline & $\%$ & 0 & 100 & 100 & 0 & 100 & 100 \\
\hline \multirow{2}{*}{ Autres } & Nbre & 0 & 8 & 8 & ") & 20 & 20 \\
\hline & $\%$ & 0 & 100 & 100 & 0 & 100 & 100 \\
\hline
\end{tabular}


$2^{\circ}$ Les espèces liées préférentiellement au bois altéré : $F$, annosus, $A$, sarcoides ;

$3^{\circ}$ Les espèces liées exclusivement au bois altéré : Scylalidium SP, autres.

c) Répartition territoriale dans l'bôte,

L'existence de ces trois groupes et la disposition ascendante de l'altération dans les arbres nous amènent à envisager l'existence d'une certaine répartition territoriale des espèces à l'intérieur de l'hôte. Cependant, les données recueillies à $0,30 \mathrm{~m}$ puis à $\mathrm{I}, 30 \mathrm{~m}$ ne permettent pas d'étudier cette répartition de façon satisfaisante puisque dans la plupart des cas étudiés, l'altération et la microflore associées dépassent le niveau $\mathrm{I}, 30 \mathrm{~m}$. L'étude de la répartition territoriale des espèces a donc nécessité d'effectuer des observations sur des hauteurs beaucoup plus importantes : elle n'a porté que sur quelques arbres : les numéros 16 (68), 80 (73), 89 (ro) et $99 \mathrm{~N}$ (hors de la série étudiée ici) ont été étudiés après abattage, le n* 86 (4I) a été laissé sur pied et sondé tous les ro $\mathrm{cm}$ jusqu'à $6 \mathrm{~m}$ de haut. Les répartitions observées sont représentées dans la figure $\mathrm{I}$. Lorsque plusieurs espèces sont présentes dans un mème arbre, elles ne se trouvent pas en mélange mais occupent en réalité des sites distincts séparés ou non par des zones non colonisées. Dans quelques cas, on peut noter une bonne colncidence de localisation entre l'altération et un champignon, c'est-à-dire une avance faible sinon nulle de celui-ci sur l'altération, c'est le cas pour $F$, ansosus chez le $n^{\circ}(73)$ et le $n^{\circ}(68)$ pour partic ainsi que pour A. sarcoides chez les $\mathrm{n}^{\circ}$ (10) et (73). L'avance est par contre importante dans les autres cas : $2 \mathrm{~m}$ environ pour A. sarcoides chez le $\mathrm{n}^{\circ}(4 \mathrm{I}), F$, annosus quant à lui présente une avance de $2,5 \mathrm{~m}$ chez le $n^{*}\left(4^{1}\right)$ ainsi que chez le $n^{*}$ (10) ; chez le no (68), une colonisation latérale se développe sur près de $1,5 \mathrm{~m}$ sans altération. Ces faits révèlent des dynamiques certainement très différentes du phénomène d'altération d'un arbre à l'autre.

Lorsque lon compare les territoires occupés par les diverses espèces, nous constatons que, dans un mểme arbre, ceux du $F$. annosus et de 1 'A. sarcoides sont toujours parfaitement distincts sans possibilité de passage de l'un à l'autre, de ce fait les deux espèces apparaissent comme concurrentes pour le substrat. Il n'en va pas de même pour les autres espèces. En effet, chez le n" $99 \mathrm{~N}, N$. fuckeliana var. fuckcliana occupe un territoire emboîté au-dessus de celui de $F$, annosus et largement en contact avec celui-ci, le mème phénomène se produit pour Mollisia ss. avec A. sarcoides chez le $\mathrm{n}^{\circ}(73)$ et avec $F$. anmosus chez le $\mathrm{n}^{\circ}(68)$; ces faits permettent d'envisager la possibilité d'une succession entre les espèces concernées à moins qu'il $\mathrm{y}$ ait antagonisme au niveau des surfaces de contact comme pourrait le laisser penser la faible progression d'A. sarcoides chez le $\mathrm{n}^{*}$ (73) et de $F$. annosus chez le $n^{\circ}(68)$.

\section{23. Sociabilité.}

Nous venons d'envisager la possibilité de concurrence et de succession entre espèces à l'intérieur des trones : plus haut, nous avons vu également que cer ain is " associations " sont possibles et que des " exclusions * sont envisageables; usus allons chercher à préciser ces phénomènes.

a) Sociabilité quantitative.

$\mathrm{Si}$, pour une espèce donnèe, nous comptons le nombre de sondages dans lesquels elle se trouve seule, puis avec une seule autre espèce, puis avec deux autres, etc, 


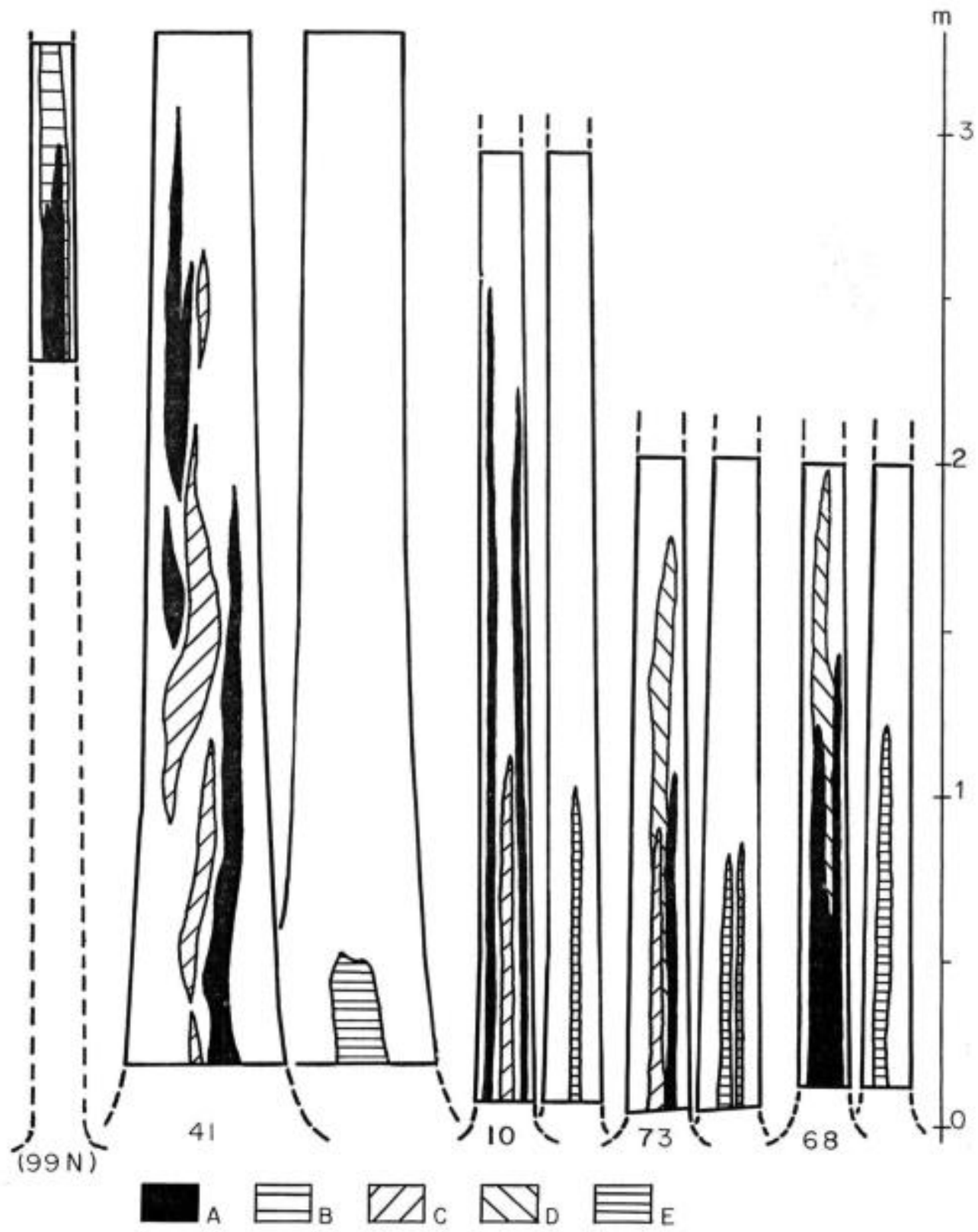

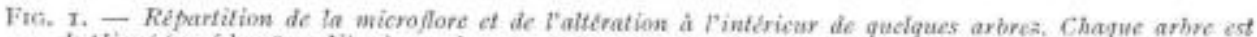
dupliqué (sauf le $n^{\circ} 99 \mathrm{~N}$ ) : à gauche est representec la microflore ot à droite lalteration.

Distribution of the microfora and of the decay in sowe trees, Every tree in duplicated (except $n^{\prime \prime} 99 \mathrm{~N}$ ) : on (he left is the microflora and on the right is the decay.

A. Fomes annosus; B. Nectria fuckeliana vak. fuckeliana; C. Ascacoryne sarvoides; D. Mollisia sp.; E. Altération du bois (Dichy). 
nous pourrons calculer sa fréquence d'spparition dans des populations de plus en plus nombreuses; l'ensemble de ces fréquences caractérise la sociabilité quantitative de l'espèce. Par convention, nous avons considéré que lorsqu'une espèce est isolée seule, il s'agit d'une population d'effectif $I$. Les nombres et fréquences obtenus de cette manière figurent au tableau 4. Dans ce tableau, lorsque l'on considère la répartition générale des populations en fonction de leur effectif, on constate qu'à $\mathrm{I}, 30 \mathrm{~m}$ les effectifs I et 2 sont largement dominants et en nombres sensiblement égaux et qu'à o,3o m les populations d'effectif 3 augmentent nettement au détriment, semble-t-il, des populations d'effectif 2 .

\section{TABI,EAU +}

Sociabilite quantitarive des espèces

Quantitatize seciability of the species

\begin{tabular}{|c|c|c|c|c|c|c|c|c|c|c|}
\hline \multirow{3}{*}{$\begin{array}{l}\text { Effectif des populations } \\
\text { (Sise of populations) }\end{array}$} & \multicolumn{8}{|c|}{$\begin{array}{l}\text { Participation des evplees aux diverses populations } \\
\text { (Contribution of the spectes to the differcml populations) }\end{array}$} & \multirow{2}{*}{\multicolumn{2}{|c|}{ Torat }} \\
\hline & \multicolumn{2}{|c|}{1} & \multicolumn{2}{|c|}{ 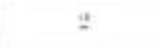 } & \multicolumn{2}{|c|}{3} & \multicolumn{2}{|c|}{4} & & \\
\hline & Nbre & $\%$ & Nbre & $\%$ & Nbre & $\%$ & Nbre: & $\%$ & Nbre & $\%$ \\
\hline 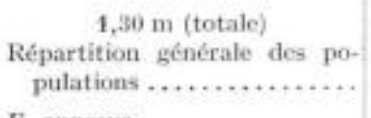 & 60 & 17,6 & i1 & $4 x, 8$ & \pm & 2,6 & 1 & 1,2 & *4 & 100 \\
\hline F. annosus, ............... & $2 x$ & 41,7 & 36 & $3 ;, 7$ & 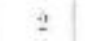 & 2,9 & 1 & 1,7 & 67 & (IN) \\
\hline Mollisia sp. ............... & 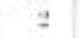 & 6,6 & 97 & 87,0 & 1 & 4,2 & 1 & 3,2 & 31 & ten \\
\hline N. fuckeliana ............. & 2 & 14,2 & 11 & 78,5 & 1 & 7,3 & 0 & 0 & 18 & 100 \\
\hline A. sarcoides $\ldots . . . \ldots+\ldots$ & 5 & 50,0 & $\beth$ & 20,0 & 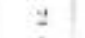 & 20,0 & 1 & 10,0 & 10 & 100 \\
\hline Scytalidium sp. ............. & 1 & 25,0 & 1 & 25,0 & 1 & 25,0 & 1 & 25,0 & 4 & 100 \\
\hline Autres (others) .............. & 2 & $\pm 5,0$ & 5 & $6:, 6$ & 1 & $1 \geq, 5$ & 0 & 0 & 8 & 100 \\
\hline $\begin{array}{l}\quad 1,30 \mathrm{~m} \text { (complète) } \\
\text { Répartition générale des po- } \\
\text { pulations } \ldots \ldots \ldots \ldots \ldots \ldots \ldots\end{array}$ & 15 & 12,8 & 17 & 48,5 & 2 & 5,8 & 1 & 2,9 & A5. & 100 \\
\hline$F$. annosers................ & 5 & 26,5 & 12 & $6: 2,1$ & 1 & 5,2 & 1 & 5,2 & (1) & 100 \\
\hline Mollisia sp. $\ldots \ldots \ldots \ldots \ldots$ & $\stackrel{2}{2}$ & 25,0 & 5 & 50,0 & 1 & 12,5 & 1 & $1 \geq, 5$ & $x$ & lan \\
\hline N. fuckeliana , , ........... & 2 & 15,6 & 11 & 83,6 & 1) & 0 & 0 & 0 & 13 & $1(4)$ \\
\hline A. sarcoides $\ldots \ldots \ldots \ldots \ldots$ & 4 & $A t, t$ & $z$ & $z \cdot 2,2$ & $\Rightarrow$ & $\pm 2,2$ & 1 & 11,2 & 4 & 10: \\
\hline Scytalidium sp. ........... & 1 & 누, 0 & 1 & 45,0 & 1 & $\pm 5,0$ & 1 & 25,0 & 6 & 100 \\
\hline Autres (others) .............. & 1 & 16,7 & 4 & 66,6 & 1 & 16,7 & 0 & 0 & 6 & 100 \\
\hline $\begin{array}{l}\quad 0,30 \mathrm{~m} \\
\text { Répartition générale des po- } \\
\text { pulations ................ }\end{array}$ & 16 & 51,2 & $1 \pm$ & 20,7 & 10 & 45,4 & 1 & 2.5 & 39 & 100 \\
\hline 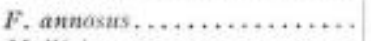 & 8 & 38,7 & $x$ & 28,7 & 6 & $\$ 6,0$ & 1 & 4,6 & 23 & 100 \\
\hline Mollisia sp. $\ldots \ldots \ldots \ldots \ldots$ & 1 & $\$ 5,0$ & $z$ & 50,0 & 1 & 25,6 & $\theta$ & 0 & 4 & 100 \\
\hline N. fuckdiana ............. & 1 & $\pm 0,0$ & \pm & 50,0 & 2 & 50,0 & 0 & (1) & a & 100 \\
\hline A. sarcoides .............. & 4 & a:t,is & 5 & 29,4 & 7 & $\$ i, t$ & 1 & 6,6 & 17 & 100 \\
\hline Scytalidium sp. ........... & 0 & 6 & 2 & 40,0 & 3 & 60,0 & 0 & 0 & 5 & 100 \\
\hline Autres (others) ........... & $z$ & 10,0 & 5 & 25,0 & 11 & 56,0 & 2 & 10,0 & 20 & 100 \\
\hline
\end{tabular}

Les chiffres en italique sont les fréquences maximales de chaque espèce.

(The underlined numbers are the maximum frequencies of exer spocies). 
Voulant analyser le comportement de chacune des espèces, nous devons d'abord remarquer que certaines d'entre elles sont trop peu abondantes pour que leur répartition en quatre catégories soit interprétable; nous nous limiterons aux plus nombreuses. A I,30 $\mathrm{m}$ (série totale), le $F$, antosus a une répartition semblable à la répartition générale, ce qui est normal puisqu'il s'agit de l'espèce dominante ; les autres s'en écartent notablement: Mollisia sP. at N. fuckeliana var. fuckeliana sont préférentiellement présentes dans des populations d'effectif 2.

A $0,30 \mathrm{~m}$, le $F$. annosus présente toujours les mêmes tendances que la répartition générale à ce niveau mais deux espèces dominent nettement dans les populations d'effectif $3:$ A. sarcoides et la catégorie \& autres $x$.

Il ressort done :

$\mathrm{I}^{\circ}$ que lorsque l'on passe du niveau $\mathrm{I}, 30 \mathrm{~m}$ au niveau $0,30 \mathrm{~m}$, nous allons vers une complexité croissante des populations de micro-organismes,

$2^{\circ}$ comparées au $F$. annosus, certaines espèces sont caractéristiques des populations simples ( 2 constituants), d'autres de populations plus complexes ( 3 constituants). Ces faits confirment l'hypothèse d'un phénomène de successions que nous pouvons chercher à préciser encore en analysant la compatibilité des espèces entre elles.

\section{b) Sociabilité qualitative.}

Si nous comptons le nombre de sondages dans lesquels une espèce A est présente avec l'espèce B puis avec chaque espèce successivement, nous pourrons calculer les fréquences de présence conjointe de l'espèce $\mathrm{A}$ avec toutes les autres prises individuellement: l'cnsemble de ces fréquences caractérisera la sociabilité qualitative de l'espèce A. Elle correspond à la notion de sociabilité développée par THIERcEI,IN ef $a l$. ( 1972 ). Les fréquences obtenues de cette manière figurent au tableau 5 ; par convention, lorsqu'une espèce est isolée seule, nous l'avons considéré associée avec elle-mème: d'autre part, connaissant le nombre de cas d'association de chaque espèce, nous avons calculé la fréquence théorique des associations deux à deux dans 1'hypothèse d'une répartition au hasard. Nous avons done à $1,30 \mathrm{~m}$ ainsi qu'à $0,30 \mathrm{~m}$ deux répartitions dont la question est de savoir si elles sont différentes ou non. Le test $\chi^{2}$ nous indique qu'à $\mathrm{r}, 30 \mathrm{~m}$ la différence est significative à I $\mathrm{P}$. Ioo mais à $0,30 \mathrm{~m}$ à $20 \mathrm{p}$. Ioo seulement. On peut done dire qu'à $\mathrm{I}, 30 \mathrm{~m}$ au moins certains couples ne se forment pas au hasard mais; compte tenu des efiectifs, il n'est pas possible de procéder à l'analyse statistique pour chaque couple. Nous pouvons néanmoins dégager quelques tendances au niveau $\mathrm{I}, 30 \mathrm{~m}$. Pour ce qui concerne le $F$, asnosus il forme des couples anormalement fréquents avec Mollisia SP. et $N$. fuckeliana confirmant sur l'ensemble des sondages à ce niveau le comportement particulier de ces deux espèces vis-à-vis du $F$, annostrs; il forme des couples anormalement peu nombreux avec $A$. sarcoides confirmant son caractère concurrent vis-à-vis du F. annosus (mais cette tendance est absente à $0,30 \mathrm{~m}$ ) ; avec la catégorie * autres o il forme des couples de fréquence normale signifiant qu'il y a indifférence. Mollisia SP, seul, apparait à $1,30 \mathrm{~m}$ avec une fréquence très faible qui est sans doute la conséquence de son association privilégiéc avec le $F$, annosus. 


\section{TABLEAT 5}

\section{Sociabilitét qualitative}

A $\mathrm{r}, 30 \mathrm{~m}$ (série totale) comme à $0,30 \mathrm{~m}$, tous les couples posaibles ont été considérés. ce qui nous conduit it 150 et 100 couples respectivement

\section{Qualitatioe sociability}

At $1.30 \mathrm{~m}$ (total series) and at $0.30 \mathrm{~m}$ every possible pair was considered, we have respectively 1,50 and 100 pairs

\begin{tabular}{|c|c|c|c|c|c|c|c|}
\hline $\begin{array}{l}1,20 \mathrm{~min} \\
\text { (totake) }\end{array}$ & $F$, anmoszs & Aollisia sp. & $\begin{array}{l}\text { X. fuckeliasma } \\
\text { vale, fuckntiana }\end{array}$ & A. sarcoides & $\begin{array}{l}\text { Scyialidium } \\
\text { sp. }\end{array}$ & Autres & Total. \\
\hline $\begin{array}{l}\text { F. annosas } \\
\text { Mollisia sp. } \\
\text { N. fuckiliand } \\
\text { vak. fuckiliana } \\
\text { A. sarcoides } \\
\text { Scylalidium sP. } \\
\text { Autres }\end{array}$ & $28 \quad 32,68$ & $\begin{array}{r}25 \\
2 \quad 16,34 \\
\quad 8,18\end{array}$ & $\begin{array}{l}7,0 \\
3,5 \\
1,5\end{array}$ & $\begin{array}{ll}2 & 6,53 \\
2 & 3,26 \\
1 & 1,4 \\
5 & 1,42\end{array}$ & $\begin{array}{ll}1 & 3,26 \\
2 & 1,667 \\
0 & 0,7 \\
3 & 0,65 \\
1 & 0,35\end{array}$ & $\begin{array}{l}4,4,2 \\
2^{4,4} \\
0^{2,4} \\
1^{0,9} \\
0,84 \\
0,42 \\
0,34\end{array}$ & $\begin{array}{l}70 \\
35 \\
15 \\
15 \\
7\end{array}$ \\
\hline $0,30 \mathrm{~m}$ & & & & & & & 150 \\
\hline $\begin{array}{l}\text { F. annosurs } \\
\text { Mollisia sp. } \\
\text { N. fuckeliana } \\
\text { vak. fuckdiana } \\
\text { A. sarcoides } \\
\text { Scytalidius si. } \\
\text { Autres }\end{array}$ & 8,61 & $\begin{array}{ll}3 & 1,55 \\
1 & 0,25\end{array}$ & $\begin{array}{l}2,17 \\
0,35 \\
0,39\end{array}$ & $\begin{array}{ll}9 & 8,06 \\
1 & 1,3 \\
\text { (1) } & 1,89 \\
\text { 6 } & 6,76\end{array}$ & $\begin{array}{l}2,48 \\
0,6 \\
0,56 \\
2,08 \\
0,66\end{array}$ & $\begin{array}{c}7^{7} 7,13 \\
4^{7,15} \\
8^{8,61} \\
3^{5,98} \\
2^{1,83}\end{array}$ & $\begin{array}{l}31 \\
5 \\
7 \\
86 \\
8 \\
=4\end{array}$ \\
\hline & & & & & & & 100 \\
\hline
\end{tabular}

Dans chaque case, le premicr chiffre est le nombre observé, le second le nombre théorique calculé suivant une répartition au hasard.

(In every case, the first number is the obserwed one, the second is the theorical one, calculated according to a randound distribution.)

\section{DISCUSSION - CONCIUSION}

Pour mettre en évidence la microflore interne des troncs d'Épicéa, nous avons utilisé une méthode et des techniques dont il convient d'analyser l'efficacité.

Le sondage à la tarière, méthode non destructive de prélèvement, ne constitue 
qn'une prise d'échantillon orientée dans un milieu rarement symétrique (zones de colonisation, altérations, cour de l'arbre : excentrés) de telle sorte qu'un échantillon donné peut ne pas être entièrement représentatif du niveau de prélèvement, cependant, à l'échelle de la placette, il y a compensation entre tous les arbres mais une certaine imprécision subsistera lorsque nous examinerons les liaisons entre espèces.

Le niveau $1,30 \mathrm{~m}$, hauteur maximum des prélèvements (hauteur d'homme), n'est certainement pas idéal dans le cas présent pour étudier les premiers stades de colonisation des trones; c'est pour cela que, chez quelques arbres, nous avons été amené à effectuer des observations à de plus grandes hauteurs mettant en cuvre des moyens plus importants (grimpage ou abattage) ; au niveau $1,30 \mathrm{~m}$, nous avons cependant recueilli des informations intéressantes sur la microflore colonisatrice primaire, confirmées et précisées par les observations effectuées plus haut.

La mise en évidence proprement dite des éléments de la microflore a été faite par incubation des carottes-échantillons sur milieu nutritif gélosé. On peut penser que la compétition entre espèces au moment de la colonisation de ce substrat peut aboutir à l'élimination de certaines d'entre elles. Cette éventualité, dans le cas présent, est réduite dans la mesure où les espèces occupent au départ un certain volume de bois dans les carottes-échantillons; de ce fait, même si elles se font supplanter au niveau du milieu nutritif, elles pourront se maintenir un certain temps et être observées au niveau de la carotte elle-même. Il reste cependant probable que certains micro-organismes ne s'expriment pas et notamment des bactéries, ceci doit être particulièrement vrai pour les prélèvements effectués en zones très altérées où le bois est souvent réduit en charpie et les micro-organismes nombreux. Ayant porté notre attention en priorité sur les pionniers de la colonisation, nous n'avons pas cherché à affiner la méthode d'isolement à ce niveau.

Les résultats que nous avons obtenus montrent que le $F$. annosus est le principal responsable de la pourriture de cour des épicéas dans la placette étudiée et nous avons pu retrouver les différents stades de l'attaque conformément au schéma classique de développement de ce champignon dans les troncs. Il est apparu également qu'avec le $F$. anuosus et à sa suite se développent un certain nombre de microorganismes mais notre but n'a pas été de préciser ce phénomène de successions au niveau du bois altéré.

Nous avons donc recherché plus particulièrement les colonisateurs primaires du bois ; $F$. annosus figure parmi ceux-ci mais nous avons trouvé également Mollisia SP. et $N$. fuckeliana var, fuckeliana dont nous avons vu qu'ils se présentent de façon telle dans les troncs et par rapport au $F$, annosus que nous devons envisager les relations qu'ils peuvent entretenir avec lui. Lorsque le $F$, annosus progresse en hauteur et qu'il rencontre un territoire déjà occupé par l'une de ces deux espèces, il peut se trouver dans trois situations différentes :

I. Il est indifférent à cette présence et sa progression n'est pas affectée, dans ce cas, il y a succession fortuite;

2. Il rencontre un territoire modifié en sa faveur où il peut progresser plus aisément et dans ce cas, il y a une succession vraie ;

3. Il est gêné par l'occupation préalable du site, par sa modification ou par une activité antagoniste et dans ce cas, il y a ralentissement ou arrêt de la progression. 
Si l'on considère le cas du Mollisia SP. son association préférentielle avec le $F$. annosus militerait en faveur de l'hypothèse de la succession vraie, de plus, la rareté des cas où il se trouve seul dans le trone laisse penser que lorsqu'il colonise un arbre, le $F$. annosus lui succède dans la plupart des cas; chez l'arbre n* (68) (fig. I) cependant on peut envisager un effet antagoniste du Mollisia sp. puisqu'il y a arrêt du $F$. annosus; nous ne pouvons donc pas conclure de façon définitive, de plus, aucune observation antérieure concernant cette espèce ne peut nous servir de référence.

Pour le $N$. fuckeliana var. fuckeliana, nos observations font apparaitre un comportement assez semblable. Des mentions antérieures de cette espèce existent dans la littérature de telle sorte qu'elle peut être considérée comme classique dans le bois de l'Épicéa sur pied. Eille a été signalée associée ou non à une altération du bois (RoI,I,-HANsen, I962 ; SchöNHAR, I969, I973; SHA1N, I971 ; BAzZIGHER, I973 ; Pechmann et Aursess, 1974). Rol.t-Hansis (I062) qui l'a étudiée spécialement signale que sa croissance peut être de $180 \mathrm{~cm}$ en 7 ans dans le tronc et les racines et qu'elle provoque tout au plus une légère coloration brun-rouge du bois sans affecter de façon apparente ses qualités. Seul SHarx (r97I) a discuté la signification de sa présence vis-à-vis du $F$. annosus : il l'isole fréquenment dans la * zone de réaction n qui se développe dans l'aubier autour de la colonne de $F$. anuosus et suggère qu'elle participe au caractère inhibiteur de cette zone. La localisation de N. fuckliana var. fuckeliana indiquée par SHAIN ( $\mathrm{I} 97 \mathrm{I}$ ) correspond bien à nos observations, cependant, cette localisation dans la * zone de réaction * n'est pas décisive pour conclure à un effet inhibiteur; en effet, et ceci est également vrai pour Mollisia sp, elle pourrait n'être que le résultat de l'élimination par $F$, annosus : lorsque celui-ci progresse, les espèces pré-existantes seraient reléguées en périphérie (cône emboité) et ne se maintiendraient dans l'aubier que parce qu'il est moins favorable au $F$. annosus.

En ce qui concerne $1^{\prime} A$, sarcoides, nous avons vu qu'il peut se comporter en colonisateur primaire des troncs (présence dans le bois sain) mais qu'on le rencontre dans la majorité des cas au niveau de zones altérées, en cela son comportement ressemble à celui de $F$, annosus.

Ce comportement a déjà été observé antérieurement; 1 'A. sarcoides a été décelé en bordure des altérations ou même dans des zones totalement saines chez Picea abies (KATo, I967; RICARD, I970), chez P. glauca, P. engelmanni, $P$. mariana (Etmeridge et Carmirhaei,, r955), P. mariana (Basham, r973) et chez Pinus contorta (Bourchrer, I96r). Il a été également décelé au niveau du bois altéré chez

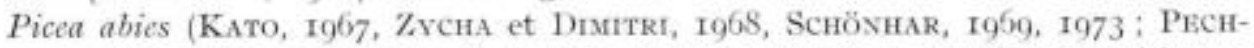
MANN et AUrsess, I97 I), chez P. mariana (BAshan, 1973) et chez d'autres résineux, notamment Pinus contorla (Nordix el $a l$., I955 ; ETHERIDGE et CARMrCHAII, I955 ;

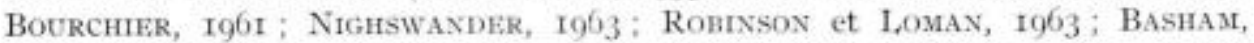
1966).

FTHERIDGE (I97o) rapporte l'observation suivante résultant d'une prospection réalisée sur Picea glauca, $P$. engelmanni et $P$. mariana en Alberta : "L'A. sarcoides était présent dans 7,1 p. 100 des échantillons provenant d'arbres sans altération ; dans 17,8 p. 1oo des échantillons apparemment sains provenant d'arbres altérés ; dans 57,1 p. roo des échantillons présentant une coloration rouge et dans environ 14 p. Ioo des échantillons présentant une pourriture commençante ou avancée. Ces faits indiquent clairement que l'A. sarcoides précède plutôt qu'il ne suit l'infection des agents d'altération confirmant son rôle d'envahisseur primaire des arbres vivants s. 
Ces observations sont confirmées par BAsham (I973) qui précise que Picea mariana n'est abondamment colonisé par ce champignon qu'à partir de 75 ans enviton. Nos observations cependant tendent à montrer que $I^{\prime} A$. sarcoides n'entretient pas avec le $F$, anuosus des relations qui entrent dans le cadre d'une succession et qu'en cela, il ne précède pas le $F$, annosus mais rentre plutôt en concurrence avec lui pour la colonisation du substrat.

D’autre part, $1^{\prime} A$. sarcoides est généralement considéré comme un champignon non lignivore compte tenu d'observations de laboratoire dont nous discuterons dans un article ultérieur. Cependant, l'observation des arbres $n^{\circ}$ (ro) et (73) milite en faveur d'une thèse inverse puisqu'il y a parfaite coïncidence entre $1^{\prime} A$. sarcoides et l'altération à moins que 1'on imagine que 1'A. sarcoides ait supplanté tctalement le vrai responsable de l'altération observée. Eñfin, il est à noter que contrairement au $F$. annosus, $1^{\prime} A$. sarcoides voit sa fréquence augmenter lorsque l'on passe du niveau $1,30 \mathrm{~m}$ à celui du pied (tabl. 2 ) indiquant au moins qu'il n'est affecté ni par l'arrivée des espèces secondaires, ni par l'état avancé de l'altération du bois.

En conclusion, nous pouvons dire que les colonisateurs primaires que nous avons mis en évidence dans le tronc de Picea abies présentent des comportements bien différents. Le $F$, annosus est un agent d'altération bien connu dont nous avons vu qu'il ouvre la voie à un cortège d'organismes secondaires dans le bois qu'il a colonisé, leur cédant la place progressivement. $\mathrm{L}$ :A. sarcoides apparait comme un concurrent du $F$, annosus pour la colonisation du substrat, leurs relations ne rentrant pas dans le cadre d'une succession; contrairement au $F$. annosus, l'A. sarcoides peut se maintenir au niveau des altérations avancées, ce qui est remarquable pour un colonisateur primaire; son rôle lignivore quoique possible ne peut pas être affirmé à partir de nos observations, il a d'ailleurs été nié par nombre d'auteurs. Quant au Mollisia sp. et at $N$. fuckeliana var. fuckeliana, ils ont des compcrtements voisins, ils sont présents surtout dans le bois sain et ne se maintiennent que rarement dans le bois altéré, leur pouvoir lignivore fait done question, de plus, lorsqu'ils sont présents en même temps que le $F$. annosus dans les arbres, ils occupent des sites situés en avant de son front de progression et largement en contact avec lui, la signification de ce voisinage a été discutée mais plusieurs hypothèses demeurent envisageables.

En fait, seul un travail expérimental pourra permettre de répondre aux questions concernant le pouvoir lignivore des colonisateurs primaires des trones et les relations que ces espèces entretiennent entre elles.

Regu pour publication wn avil 1976.

\section{REMERCIEMENTS}

Nous tenons à remercier :

- 16.N.F., Centre de Neufchâteau et la commune de Liffol-le-Grand pour les facilités de travail en forèt,

- M. J. Ricard qui est à l'origine de ce travail,

- M. le Professeur Bombs et M. Berrher pour l'envoi de souches du genre Ascocoryne.

- MM. Proxos et Permis pour leur aide de tons les jours ainsi que M. Mokelet plus particulièrement pour son aicle en matière de systématique,

- Mme Gilberte Sylvestre, épouse Gusot dont le nom figure en tête de l'article pour son indispensable aide technique. 


\title{
SUMMARY
}

\author{
INTTENAI, MICROHLORA OF THE WOOD TISSUS \\ OF THE STANDING NORWAY SPRUCE \\ I. - INVENTORY CF THE NATURAL, MICROFLORA
}

With the ultimate aim of studying its influence on the propagation of $F$. anwosus inside trees (Picea abies), the author studied the associated microflora of $F$. annosus. 100 trees were sampled at $1,30 \mathrm{~m}$ level and 49 of them at $0.30 \mathrm{~m} ; 5$ trees were then felled for more accurate observations. The study of the standing trees was made with the Pressler borer; by putting the cores on a nutritive medium the micro-organisms were obtained. In addition to $F$, ammosus we found: Mollisia sp. Nectria juckeliana var. fuckeliama, Ascocoryne sarcoides and less frequently, fungi of the genus Scylalidium, Trichoderma, mucorales, bacteries.

According to their position in the sound or decayed wood, three groups were able to be distinguished :

1. The species located exclusively in the decayed wood (Scytalidium, Trichoderma, bacteries),

2. The species located preferentialy in the sound wood (Mollisia sip., $N$. fuckeliana var. fuckeliana). coides).

3. The species located indifferently in the sound or the decayed woot $(F$-annosus, $A$, sar-

The group $\mathrm{r}$, more abundant at the $0.30 \mathrm{~m}$ level is typical of advancexl stages of the decay in which $F$, amuosus may be eliminated. The group 2 is composed by fungi which seem to precede $F$. anuosus but is it not possible to define their atction only by observation of the natural phenomenon. The presence of Mollisia sp. here is an original observation. The group 3 is that of the agents of decay; this is well known for $F$, annosus but rests under discussion for $A$, sarcoides and needs to be confirmed.

\section{RÉFÉRENCES BIBI,IOGRAPHIQUESS}

Anoxvme, 1924. La pourriture rouge de l'lepicta en Belsique. Soe. Cent. Forest. de Bdg. But., 31 , $653-657$.

Basnam J. T., 1966 . Heart rot of jack pine in Ontario. I. The occurrence of basidionysetes and micno: fungi in defective and normal heart-wood of living jack pine. Can J. Bot, 44, 275-295.

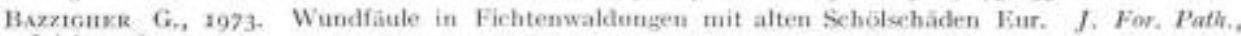
$3(2), 71-82$.

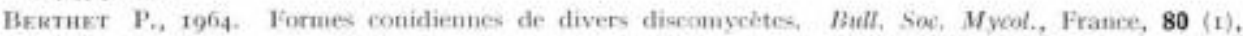
125:149.

Boorн C., 1966. The senus Cylindracarpon, Myc, Pap., 101, 1-56.

Bovecus:R R. J., 1965. Laboratory stucties in microfungi isolated from the stems of living lodgepole pine, Pinus contorla dotach. Can. I. Bet., 39, $1373+43^{4} 3$.

Detatove C., T972. Le Fomes anuosas. R. F, F., 21 (1), 20-38.

linusutec D. F., to53. Deray of subalpine spruce on the rocky mountains forest reverves in Alberta. Bi-m, Progr, Kep, Dept. A zrie. Can., 9 (6), 3 .

ВтнекарGe D.. 13, 1956, Oceurrence of Coryne sarcoides with heartrot funzi on spruce in Alberta, Canada. Absty. Bril. Mycod. Soc. Trans., 39 (3), 385-386.

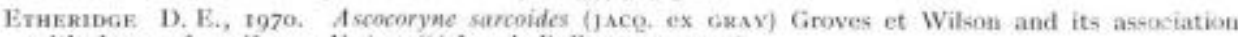
with decay of conifen, Uniwrsitd Javal. Bull, $\mathrm{n}^{0} 13,19-26$.

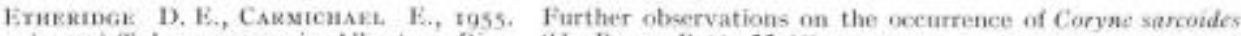
(jacg.) Tul, on spruce in Alberta. Bi-monthly Progr. Rept., 11 (6), 3 .

Като F., 1967. Anftreten und Bedeutung des Wurzelschwanmes (Foars anasas (Fr.) cooks) in Fichtenbeständen Niedersiachsenx, Schriftenveibe Forstl. Fak. V'nin, Gülingen, 39, 35-120.

Kersostrost A., Bever L., 1965. Two new species of Scyalidium with antagonistic properties for Fowes ammosars. Stchsk, Bot. Tids, 59, 30. 
Le Gal. M., Maxersor F., Contribution à l'étude des Mollisioidecs.

5958. Rewuc de Mycologic, 23, 28-86,

1960. Revue de Myoologie, 25, 135-214.

1965. Revue de Mycologic, 26, 262-33r.

1966. Revue de Mycologie, 31, 3-44.

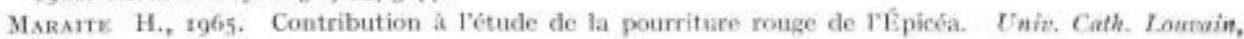
$82 \mathrm{p}$.

Nrohswander J. E., 1963 . Record of occurrence of Coryne sarcoides in lodsepole pine and association with decay fungi. In Z Ann. Rept. For. Entomology and Pathology Branch, Depe. Forestry, Cantitia year ending march.

Nokdix V. J., Hextse W. O., Berru W., r953. Red stain and other decays of lodgepole pine in Alberta, Interim Rept. 1955-1. For. Biol. Lab., Calgary, Alta, april 1955.

Pawsry R. G. Stankovicovs L., 1974. Studies of extraction damage decay in crops of Picea abies in Southern England. I. Examination of crops dauaged during uormal forest operations. Eur. J. For. Path., 4, 1 $29-137$,

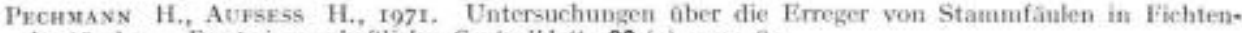
beständen, Forstwissenschaftliches Centralbatt., $90(4), 259-284$.

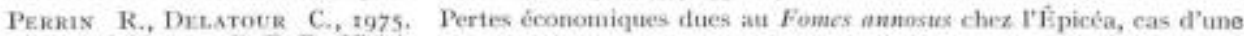
coupe a blanc, $R, F, F, 27(3), 192-195$.

Peknis R., Delayouat C. Méthode d'estimation de la hauteur de pourriture dans le trone des lipictas sur pied attaqués par le Fomes annosis (rk.) cooks. Rur. J. For. Path., (sous presse).

Pesaxte A, 1956, Osservazioni st una carie del Platano. Ann. Sper. Agr. N. S, 11, z49-266.

ReNszikvelt B., 1947. Om rotrotan (Polyporus annesus rk.) i Svorige. Deiss utbreding och sâtt att upptrida? Modd. Skogsfonsk. Inst. Stockh., 35, r-88,

Rucaub J., r97o, Biological control of Fones anusues in Norway spruce (Piced abies) with imumnizing commensals. Studia forestalia sirecica, 84, 50 p.

Ricard J., I975. Biological control of decay in Denulas fir poles, Seven years perspective. Eiur. $I$. For, Path, 5, 175-177.

Ronrssox R. C., Loxsx A. A., 1963. Fungi isolated in culture from red heartwood stain and advan. ced decay of lodgepole pine in Alberta, Can, J. Lat, 41, 137r-1375.

Rot.t-HaxseN F, 1962. Nectria cucurbifala sensis Wollenweber, its Cephabosporium state and some other Cephalosperiam siv. from stems of conifers. Meddelelser fra Det norshe skorforsokstersen, 27 (3), $293-3+2$.

Sсно̆хнх S., I969. Untersuchumgen über das Vorkotnmen von Rotfiulepilzen in Fichtenbestanden der Schwibischen Alb. Milteilungen des Vereins fiir Forsthiche Standortskunde und Forstpjansensüchfung, 19, $20-28$.

Scnöxhar S., 1973. Zur Ausbreitung yon Fones annosss und anderer Rotfanlepilxe in Fichtenbestitnden 2. jeneration. Mitteilungen des Vervins fir Forstliche Standortskunde und Forst pjlansenssichtung, 22, 3-8,

Sirsin L., 1971. The response of sapwood of Norway spruce to infection by fontes annasas, Phytopath, $61(3), 301-307$ -

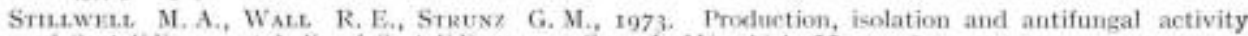
of Scyfalidin a metabolic of Scytalidium sr. Can. J. Microbial, 19, 597-60z.

Srrusz G. M., КАкиsumo M., Sriblwzl. M. A., 1972. Scyalidin : a new fungitoxic metabolite produoed by Scytalidizm sp. I. CAcm. Soc., 18, 2280-2283.

Svivestre. G., 1974. Au sujet de la désinfection des caroffes prideves à la tariáre de Pressfer. C.N.R.F. Laboratoire de Pathologie forestière, Distribution limitée, document $n^{\circ} 74 / 23 \mathrm{P}$.

Tuzucelax F., Arsould M.-F., Mangenot 1\%, Potok H., 1972. Altérations du bois provoquécs par les sondages à la tarière, Leur contríle, Ann, Sci, for, 29 (1), 107-133.

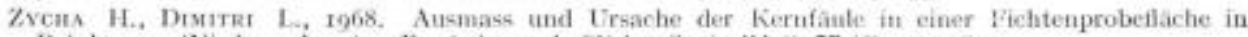
Reinhausen (Niedersachen), Forsteissenschafliches Contralblath, 87 (6), 321-384. 